\title{
Analyse De La Promotion Du Genre Et De L'équité Dans Le Processus Du Développement Local : Cas Du PDC De La Commune De Matamèye
}

\author{
Zakari Aboubacar \\ Maître-assistant, Sociologue, Département de Sociologie, \\ Faculté des Lettres et Sciences Humaines, Université de Zinder, Niger
}

Doi: 10.19044/esj.2017.v13n29p254 URL:http://dx.doi.org/10.19044/esj.2017.v13n29p254

\begin{abstract}
This study aims is to identify the level of integration of the female component in development actions at the level of the urban commune of Matamèye. It is based on an exploitation of the field data with the help of a maintenance guide, a questionnaire and the support of the Municipal Development Plan. The interview took place in the semi-structured form. It is clear that there are more than a hundred registered women's groups both in the commune and in the departmental service. These groups operate in the following activities: extraction of peanut oil, market gardening, manufacture of traditional spaghetti and garin yara. The promoters of these activities do not complain. Indeed, even if these results do not live up to expectations, we must encourage the initiatives of the municipality which try with little means to create a framework that allows these structures to evolve in complete independence. Nevertheless, there are conflicts of jurisdiction in the granting of official authorization to these groups between the supervisory authority and the newly elected administration. Each of the two parties believes that it is up to the municipality to issue them according to the local authorities code according to the ordinance $n{ }^{\circ} 2010-54$ of 17 September 2010. That is why we say that the conflicts of competence between the supervisory authority and the newly elected government that often appear must not hamper local development actions, the principle for which decentralization has emerged.
\end{abstract}

Keywords: Decentralization, local development, gender promotion, equity, communal development plan

\section{Résumé}

Cette étude a pour objectif de cerner le niveau d'intégration de la composante féminine dans les actions de développement au niveau de la commune urbaine de Matamèye. Elle s'appuie sur une exploitation des 
données de terrain à l'aide d'un guide d'entretien, d'un questionnaire et avec comme support le Plan du Développement Communal de ladite commune. L'entretien s'est déroulé sous la forme semi-structurée. Il ressort clairement qu'il existe plus d'une centaine de groupements féminins enregistrés tant à la commune qu'au niveau du service départemental. Ces groupements opèrent dans des activités suivantes : extraction d'huile d'arachide, le maraichage, la fabrication des spaghettis traditionnels ainsi que le «garin yara ${ }^{70}$ ». Les promotrices de ces activités ne s'en plaignent pas. En effet, même, si ces résultats ne sont pas à la hauteur des attentes, il faut encourager les initiatives de la commune qui essaient avec peu de moyens de créer un cadre qui permet à ces structures d'évoluer en toute indépendance. Néanmoins, il existe des conflits de compétence dans l'attribution de l'agrément officiel à ces groupements entre l'autorité de tutelle et la nouvelle administration élue. Chacune des deux parties pensent que c'est à elle d'en délivrer alors que selon le code des collectivités territoriales suivant l'ordonnance n²010-54 du 17 septembre 2010, ce pouvoir revient à la commune. C'est pourquoi nous disons que les conflits de compétences entre l'autorité de tutelle et la nouvelle administration élue qui apparaissent souvent ne doivent pas entraver les actions de développement local, principe pour lequel est issue la décentralisation.

Mots-clés : Décentralisation, développement local, promotion du genre, équité, plan du développement communal

\section{Introduction}

La gestion d'une société requiert toute une organisation bien structurée. Dans les pays africains colonisés, ce système est passé du statut trouvé sur place par les colons, à celui qu'ils ont instauré avant de laisser la place aux systèmes post indépendances. A chaque niveau, c'est la participation populaire qui est recherchée. Le colon a institué l'impôt pour pouvoir soutenir les charges de la colonie. Le nouveau pouvoir ne l'a pas supprimé mais essaie de s'approcher de la population locale par des actions de développement. S'agissant du Niger, il convient de noter que la loi 64-023 du 17 juillet 1964, portant création des circonscriptions administratives et des collectivités territoriales, subdivisait le territoire de la République en départements, arrondissements et communes. Les arrondissements et les communes furent érigés en collectivités territoriales dotées de la personnalité morale et de l'autonomie financière ${ }^{71}$. Ces collectivités étaient placées sous la responsabilité des sous-préfets (arrondissements) et des maires (communes). Ce système qui sévissait jusqu'à l'adoption du système de décentralisation et

\footnotetext{
${ }^{70}$ Expression hausa signifiant farine pour les enfants malnutris.

${ }^{71}$ La loi 64-023 du 17 juillet 1964
} 
les élections locales de 2004 et 2011 pour voir réellement des élus locaux dans la gestion des affaires courantes de leurs communes. Dans cette recherche, nous menons une investigation afin de mieux comprendre l'intégration du genre dans les actions de développement au niveau de la commune urbaine de Matamèye. Il existe plus d'une centaine de groupements féminins qui évoluent dans la commune. Quelles sont les actions menées par ces groupements ? Quel bilan peut-on faire en matière d'intégration de la composante féminine dans les actions de développement définies dans le plan du développement communal ? Est-ce qu'il y a une différence dans la pratique entre l'ancienne administration et celle gérée par les élus locaux?

\section{Problématique}

Le mode de gestion des sociétés urbaines du $21^{\text {ème }}$ siècle est une question d'actualité. En effet, il est important de retenir que faire une analyse des modes de gouvernance urbaine revient à considérer les manières dont le pouvoir est exercé dans la gestion des ressources de la ville en vue de son développement. C'est pourquoi, la gouvernance urbaine favorise la mise en place de partenariats qui impliquent une Co-construction des règles, des savoirs, des projets. Elle est, de ce fait, un processus très différent d'une imposition auprès de la population (injonction) à participer à des projets définis par la puissance publique.

Ainsi, d'après les lois $\mathrm{N}^{\circ}$ 2002-014/2002-015/2002-016 du 11juin 2002 portant création des communes et fixant le nom de leurs chefs-lieux et celles $\mathrm{N}^{\circ}$ 98-30/98-031 du 14 septembre 1998, portant carte de l'administration territoriale du Niger, modifiées par l'ordonnance $\mathrm{n}^{\circ} 2010-54$ du 17 septembre 2010 portant code général des collectivités territoires, la Ville de Matamèye est une commune urbaine administrée par 13 conseillers élus et 5 conseillers membres de droit ( 3 chefs traditionnels et 2 députés nationaux $)^{72}$. Cette équipe est chargée sous la supervision du Maire d'exécuter les programmes de développement de ladite Ville ${ }^{73}$.

La commune urbaine de Matamèye est sous son deuxième mandat de mise en œuvre du processus de décentralisation. Par ailleurs, l'outil d'accompagnement des communes au Niger est leur plan de développement communal. Ainsi pour son deuxième $\mathrm{PDC}$, la commune a engagé un processus de révision dudit document. Cette révision engagée au mois de janvier 2012, a non seulement permis de faire un état de lieux de la situation socio-économique de la Commune, mais aussi et surtout de définir et dimensionner les actions à entreprendre pour améliorer les conditions de vie

\footnotetext{
72 PDC CU Matamèye (2012-2016)

${ }^{73}$ Code général des collectivités territoriales du Niger, 2011.
} 
des populations. La démarche suivie s'inscrit dans les principes directeurs contenus dans le guide national révisé. Les travaux ont été conduits par les membres du comité de révision, avec l'appui technique et méthodologique de la Direction Régionale de l'Aménagement du Territoire et du Développement Communautaire (DRAT/DC) de Zinder ${ }^{74}$. Pour faciliter la collecte de données et assurer une meilleure couverture de l'espace communal (en termes d'investigations), trois (3) centres de regroupement ont été constitués : Godo, Kirou et Matamèye.

Les principaux axes stratégiques d'intervention retenus sont :

1. L'augmentation de la production agro-sylvo-pastorale ;

2. L'accès et l'accessibilité aux services sociaux de base (Eau, Santé, Education) ;

3. La maitrise de la croissance démographique ;

4. L'amélioration de la gouvernance locale ;

Parmi les axes transversaux on note :

- la promotion du genre et de l'équité dans toutes les actions de développement ;

- la lutte contre le désœuvrement des jeunes ;

- la lutte contre les IST/VIH/SIDA.

L'évaluation budgétaire des actions à mettre en œuvre dans la période 2012 - 2016, en vue d'améliorer les conditions de vie des populations, a donné un montant total de $\mathbf{1 . 4 8 2 . 0 0 0 . 5 0 0}$ FCFA $^{75}$. Les prévisions de contributions de la commune et des communautés bénéficiaires s'élèvent respectivement à 88.899.500 FCFA et 9.655.000 FCFA $^{76}$. Le montant à mobiliser auprès des partenaires et autres contributeurs est estimé à 1.383.446.000 F CFA $^{77}$ (la SNV Zinder contribuera pour son programme «Gouvernance locale, Eau, Hygiène et Assainissement» à Hauteur de 16.128.000 FCFA, entre 2012 et 2014) ${ }^{78}$.

Dans cette recherche, l'axe privilégié est la promotion du genre et de l'équité dans toutes les actions de développement .Selon les responsables de cette commune, il existe plus de cent (100) groupements féminins qui ont tous une vocation au développement des activités socio-économiques de leurs membres de façon particulière et de façon générale à celui de la commune. Ce qui de façon organisationnelle, veut dire que toutes les composantes de la commune sont prises en compte (hommes/femmes). Mais entre la théorie et la pratique, il existe un décalage, pour ne pas dire une certaine «anomie» terme utilisé par Durkheim. Il existe certes des

\footnotetext{
${ }^{74}$ PDC CU Matamèye (2012-2016)

${ }^{75}$ PDC CU Matamèye (2012-2016)

${ }^{76}$ PDC CU Matamèye (2012-2016)

${ }^{77}$ PDC CU Matamèye (2012-2016)

${ }^{78}$ PDC CU Matamèye (2012-2016)
} 
groupements féminins, la mairie associe toutes les composantes à ses différentes actions surtout celle exécutées avec la GIZ qui intervient dans le cadre de la migration depuis 2016. A en juger de très près au niveau des acteurs concernés, il ya une sensible amélioration du cadre de vie : quelques points de vente spécialement féminins, un maraichage remis sur les rails, mais, il faut que le cadre se pérennise pour passer du simple groupement à la coopérative.

Dans cette recherche, nous nous sommes intéressés à la promotion du genre et de l'équité dans toutes les actions de développement entreprises par les groupements féminins afin de cerner le niveau d'intégration de cette composante dans les actions du développement au sein de la commune. Pour cela, nous avons interrogé non seulement les élus locaux mais aussi la population bénéficiaire. Il est question pour nous de comprendre le sens que les élus locaux donnent aux actions de développement dans leur commune, une compréhension sociologique à la wébérienne. C'est pourquoi, nous formulons la question de recherche suivante : comment peut-on expliquer la promotion du genre ainsi que la question d'équité dans toutes les actions de développement entreprises par les groupements féminins reconnus officiellement dans la commune de Matamèye? Cette question générale de la recherche renvoie à l'hypothèse générale suivante: «les actions du développement sont déterminées par la promotion et l'équité entre le genre ». L'objectif général de la recherche est de cerner le niveau d'intégration de cette composante dans les actions du développement au sein de la commune.

\section{Méthodologie de recherche}

L'objectif général de cette recherche est de cerner le niveau d'intégration de cette composante dans les actions du développement de la commune. Pour cela, une mission de pré enquête a fait le déplacement sur Matamèye du 18 au 19 juin 2017 pour une prise de contact avec les Responsables administratifs et coutumiers. Cette mission est couplée avec la recherche documentaire afin de réaliser un premier draft du questionnaire et constituer un premier échantillon de la recherche.

$\mathrm{Au}$ cours d'une deuxième mission, en plus de l'entretien avec le Maire, nous avions administré 50 questionnaires aux dix groupements (le nombre est variable) qui ont leurs actes de reconnaissance officielle au niveau de la Mairie en raison de cinq interviewés par groupement soit 50 personnes suivant la méthode de saturation du contenu parce que le nombre d'adhérent est variable en fonction des groupements. En effet, il n'ya pas un nombre fixe pour chaque groupement. C'est pourquoi nous avons opté pour la saturation du contenu. L'enquête a eu lieu du 12 au 20 Août 2017 à Matamèye. 


\section{Présentation de la zone d'étude}

La Commune Urbaine de Matamèye fait partie des neuf (9) communes, que compte le département de Kantché. Elle est créée, à l'instar de toutes les autres communes du Niger, par la loi 2002-014 du 11 Juin 2002, portant création des communes et fixant le nom de leurs chefs-lieux modifiée par l'ordonnance n²010-54 du 17 septembre 2010 portant code général des collectivités territoires qui prévoient trois types de communes. Située au centre du département de Kantché, elle est limitée au nord par les communes de Kantché et Ichirnawa, au sud par Yaouri et Tsaouni, à l'est par Doungou, et à l'ouest par Daouché. Le chef-lieu de la commune est Matamèye, situé à $87 \mathrm{~km}$ au sud-ouest de Zinder sur la RN 10. Elle couvre une superficie de $447 \mathrm{~km}^{2}$, soit $13,62 \%$ de celle du département ${ }^{79}$.

La population totale est de 59.919 habitants, dont 29.659 hommes $(49,49 \%)$ et 30.260 femmes $(50,51 \%)$ (Source projection INS 2012). La densité moyenne est de 134 habitants $/ \mathrm{km}^{2}$, relativement moins élevée que celle du département (150 habitants $/ \mathrm{km}^{2}$ ). Le taux d'accroissement est de $3,5 \%$, celui de fécondité est de $6,8 \%$. Les jeunes âgés de 15 à 45 ans représentent environ $41,71 \%{ }^{80}$.

C'est au niveau de cette commune que nous menons des recherches sur la décentralisation et le développement local avec un accent sur la promotion du genre en matière du développement local. Il convient de définir les concepts principaux afin de mieux cerner la problématique de l'étude.

\section{Définition des concepts}

\section{Décentralisation et développement local}

Il est important de souligner que dès qu'on évoque la notion de décentralisation, on pense au développement local. En effet comme le note, Jean-Christophe DEBERRE (2007), de façon assez naturelle aujourd'hui s'est installée l'évidence d'une relation transitive entre décentralisation et développement local, comme si l'une engendrait l'autre, qui trouverait en elle l'instrument logique de sa réalisation. Cette évidence détermine - autour d'un processus institutionnel en phase de généralisation dans les pays en développement, quand il n'était pas inscrit dans la formation des États nouvellement indépendants - une assez forte adhésion du partenariat international $^{81}$.

\footnotetext{
${ }^{79}$ PDC CU Matamèye (2012-2016)

${ }^{80}$ PDC CU Matamèye (2012-2016)

${ }^{81}$ Jean-Christophe DEBERRE (2007), Afrique contemporaine ( $\left.{ }^{\circ} 221\right)$, Éditeur : De Boeck Supérieur, pp 45-54.
} 
Et l'auteur d'ajouter, entre décentralisation et développement local, il y va plus que d'un accommodement entre deux modes de gestion .La décentralisation redistribue des compétences centrales vers les périphéries de l'État, le développement local tient compte de la participation à la base des forces qui composent une communauté. ${ }^{82}$ La question posée est celle d'une gouvernance qui mette à la disposition d'une collectivité nationale des outils institutionnels adaptés à des formes et organisations de la vie, déposés et travaillés par l'histoire et qui réussisse à lever les suspicions réciproques : tantôt sur les intentions de l'État décentralisateur. En définitive, telle que définie dans ses principes la décentralisation au Niger est une action de déconcentrer les actions de l'Etat central vers les autorités locales élues. En général, ces autorités établissent ce qu'on appelle un programme d'action qui doit répondre aux préoccupations des populations locales, c'est-à-dire leur développement. Dans cette recherche nous nous intéressons au niveau d'intégration de la composante féminine dans les actions de développement de la commune urbaine de Matamèye au Niger afin de constater ce qui a réellement changer dans leurs vies quotidiennes.

\section{Promotion du genre et équité}

Il est à retenir que le genre se réfère aux différences et aux relations sociales entre les hommes et les femmes. Celles-ci sont acquises et varient considérablement d'une société, d'une culture et d'une époque à l'autre. Le mot genre ne saurait remplacer celui de sexe, qui renvoie exclusivement aux différences biologiques entre hommes et femmes. Les données statistiques, par exemple, sont ventilées par sexe. L'analyse des rôles, des responsabilités, des contraintes et des besoins des femmes et des hommes dans tous les domaines dans un contexte social donné fait appel au genre. Des recherches montrent que le genre est passé du cadre de «rôle sexuel» à la reconnaissance de sa participation aux structures de pouvoir et aux relations économiques. En outre, les identités liées au genre sont multiples, divisées et potentiellement instables; le genre comprend toujours la dynamique de l'appartenance ethnique et de la classe sociale ${ }^{83}$.

L'égalité entre les genres signifie que les droits, les responsabilités des femmes et des hommes ainsi que les possibilités qui leur sont offertes ne dépendront pas du fait d'être né de l'un ou de l'autre sexe. L'égalité entre les genres est également définie sur le plan de la distribution égale du pouvoir économique et doit être comprise comme une distribution d'influence, de pouvoir et d'opportunités fondée sur la parité.

\footnotetext{
82 ibidem

${ }^{83}$ http://www.genreenaction.net
} 
Atteindre l'égalité des genres ne signifie pas que les femmes et les hommes deviendront exactement égaux. Cela veut dire plutôt que les femmes et les hommes doivent avoir la même valeur dans la société.

Pour s'accomplir, la démocratie requiert une participation et une représentation égale des citoyens des deux sexes dans l'économie et la prise de décision, ainsi que dans la vie sociale, culturelle et civile. L'équité entre les genres signifie qu'un traitement impartial doit être accordé aux hommes et aux femmes, en fonction de leurs besoins respectifs. Ce traitement peut être identique ou différent, mais il doit être équivalent en termes de droits, d'avantages, d'obligations et d'opportunités.

Dans cette recherche, il est question de constater si réellement les hommes et les femmes sont associées aux actions de développement entreprises par la commune en leurs noms et faire ressortir des cas de réussite. Ce qui nous a permis de comprendre qu'effectivement les femmes sont intégrées dans le processus du développement de cette commune.

\section{PDC 2012-2016}

Par définition le plan du développement communal PDC est un document de planification simplifiée qui définit en fonction des priorités et des ressources locales disponibles les principes d'action, d'aménagement de développement social et économique de la commune sur une période de temps $(3,4 \text { ou } 5 \text { ans })^{84}$.

Le PDC est un cadre de référence pour l'investissement dans les communes. Il s'agit donc d'un document qui en fonction de potentialité et de contrainte du milieu, des objectifs de développement constaté par le conseil municipal et la société civile d'établir un programme à cours ou au moyen terme d'investissements adoptés aux réalités locales. C'est un support élaboré par le ministre de l'aménagement du territoire et du développement communautaire pour permettre aux communes nouvellement installées de pouvoir mettre en œuvre leur plan du développement économique et social. A titre d'exemple le PDC de la commune urbaine de Matamèye pour la période de 2012-2016 a retenu les axes suivant d'interventions :

1- l'augmentation de la production agro-sylvo pastorale,

2- l'accès et l'accessibilité aux services sociaux de base (eau, santé, éducation),

3- la maitrise de la croissance démographique,

4- l'amélioration de la gouvernance locale,

5- la promotion de genre et de l'équité dans toutes les actions de développement,

\footnotetext{
${ }^{84}$ Mahaman ABA, <<processus de planification communale au Niger: démarche, acteurs impliques et difficultés ». Working paper n34-2009.
} 
6- la lutte contre le désœuvrement des jeunes,

7- la lutte contre les IST, VIH Sida.

Nous nous intéressons à l'axe relatif à la promotion de genre et de l'équité dans toutes les actions de développement.

\section{Résultats de la recherche \\ Le développement local}

Dans cette recherche, le « développement local » désigne un processus consistant à mobiliser les énergies de tous les acteurs locaux en vue de la promotion économique, sociale et culturelle d'un territoire. Autrement dit, c'est un processus qui vise la participation des acteurs avec pour finalité l'amélioration des conditions de vie des habitants d'une zone déterminée. Il est à souligner ici que les acteurs du développement à l'échelle locale sont très hétérogènes : le Maire et les conseillers municipaux, les services déconcentrés de l'Etat, le gouvernement central, les composantes de la société civile (ONG locales, associations, organisations religieuses, organisations paysannes, chefs coutumiers) et les agences de coopération (agences bilatérales et multilatérales, ONG internationales).

En effet, la problématique d'un développement « durable » se pose désormais sur des bases territoriales. En ce sens, le développement local durable est le plus souvent défini comme un processus de « diversification et d'enrichissement des activités économiques et sociales sur un territoire à partir de la mobilisation et de la coordination de ses ressources et de ses énergies». La notion de territoire est, ainsi que l'évoque Patrick Badouel ${ }^{85}$, « à géométrie variable » par rapport aux problématiques de développement.

Un territoire se définit soit par une homogénéité physique et géographique, soit par une identité socio-culturelle, ou encore par une localisation sectorielle et industrielle. Celui-ci peut se situer à différentes échelles: internationale, nationale, régionale et locale. On peut donc comprendre le territoire comme un espace organisé à différentes échelles de pertinence qui régulent les processus de décision et d'action. Il est un enjeu de l'action collective qui donne aux entreprises l'accès à des ressources stratégiques. Le territoire est un facteur de rassemblement, un épicentre fédérateur du système social.

Selon Maurice Blanc ${ }^{86}$, il existe trois niveaux en ce qui concerne la transaction locale entre des hommes et leurs ressources. Cette transaction se situe à divers niveaux: i) la régulation politique, qui envisage le développement local sous l'angle de la décentralisation et des politiques d'emploi, puis des valeurs de la concertation et de la démocratie;ii) la

\footnotetext{
${ }^{85}$ P. Badouel. Rapports ETD

${ }^{86}$ Maurice Blanc, « La transaction sociale », Ed. L'Harmattan, Paris, 1992.
} 
planification économique et la création de ressources qui la circonscrit sur un territoire géographique qui fait lien entre des acteurs, des secteurs industriels et une économie régionale ;ii) l'intervention sociologique, qui considère le développement local comme un système d'action et de création institutionnelle, voire d'identités communautaires faisant appel à une économie solidaire sur un milieu support.

Il est à souligner enfin qu'aucun développement local ne ressemble à un autre. Il n'y a pas de « recette » du développement local et chaque cas constitue une "signature » spécifique. Miser sur une reproduction ou un transfert possible semble en ce domaine aléatoire, selon ce que Coudrieux ${ }^{87}$ nomme le postulat d'indétermination: « le développement relève des interfaces, des entredeux et les connexions inter systémiques ne se font jamais de la même façon; autrement dit, les mêmes causes ne produisent jamais les mêmes effets, les situations sont et restent indéterminées. Dans tout système, plusieurs possibilités potentielles existent simultanément. Ce qui rend toute évolution imprévisible. C'est le postulat d'indétermination» ${ }^{88}$.

C'est pourquoi en ce qui concerne Matamèye, il est à souligner que les actions de développement sont mises en œuvre sur ce territoire de 447 $\mathrm{km}^{2}$ de superficie, avec un conseil municipal présidé par un Maire sous la tutelle administrative du département de Kantché. Les résultats en termes de développement local ne sont pas à négliger même si dans ce domaine, il faut se référer au cas général du pays.

Le développement tant prôné tarde à prendre le chemin du niveau local comme en témoigne notre indice de développement humain (IDH $<0,4$ selon le PNUD, 2015) ${ }^{89}$.

\section{Actions menées et bilan en matière d'intégration de la composante féminine :}

Par rapport à ces aspects, les entretiens nous ont orientés vers la Mairie qui est le cordon ombilical entre les acteurs (femmes de la commune) et les partenaires au développement. Il est à souligner que cette commune renferme assez de partenaires surtout avec le phénomène migratoire qui est d'actualité. L'entretien adressé au Maire est destiné à savoir l'orientation que la commune donne aux actions contenues dans le Plan de Développement Communal ainsi qu'une estimation des groupements

\footnotetext{
${ }^{87}$ Hubert Coudrieux, 1988(a), La science des systèmes et des exploitations agricoles, Ed. Universitaires, UNMFREO, 1990.

${ }^{88}$ Hubert Coudrieux, 1988(b), La science des systèmes et des exploitations agricoles, Ed. Universitaires, UNMFREO, 1990.

${ }^{89}$ PNUD 2015
} 
féminins exerçant des activités pour la promotion du genre et de l'équité. Concernant le nombre de groupement que comprend la commune de Matamèye, le maire n'a pas des chiffres exacts mais dit qu'en même que le nombre est important car il dépasse la centaine.

Quant au point concernant les groupements, les formations qu'ils reçoivent et les ONG qui les appuient, il nous a affirmé que la présence des ONG dans le département de Matamèye qui administre la commune du point de vue tutelle administrative, a non seulement favorisé la création d'un nombre important des groupements mais aussi la formation et la subvention de celles-ci.

S'agissant des activités que font ces groupements, le constat, selon le maire, sont qu'elles sont de diverses formes: l'extraction d'huile, de maraichage, spaghettis traditionnels, de la fabrication de "garin yara ». Il continue toujours en disant que ces activités ont beaucoup contribué à la participation de la femme dans la lutte contre la pauvreté et la promotion du genre. C'est pourquoi, selon lui, bon nombre d'ONGs de développement donnent plus d'importance à ces groupements pour vulgariser certaines technologies car selon elles, ces structures sont plus motivées.

Pour terminer ces propos, le Maire de cette commune pense que pour pérenniser ces actions qui ne sont pas négligeables, il faut non seulement assurer une formation continue de ces groupements, les sensibiliser, les doter de tous les outils dont ils ont besoin, leur donner un appui conseil, les formaliser tous mais aussi, leur faciliter l'accès au crédit auprès des institutions de micro finances.

En plus de l'entretien du maire, nous avons procédé à l'administration du questionnaire aux membres des groupements féminins sur la question de la promotion du genre à celle de l'équité et des recommandations.

A ce niveau, nous avions administré 50 questionnaires aux dix groupements qui ont leurs actes de reconnaissance officielle au niveau de la Mairie en raison de cinq interviewées par groupement suivant la méthode de saturation du contenu. Les membres interrogés affirment que «leurs groupements sont reconnus et sont soutenus par une ou plusieurs ONGs parmi lesquelles on retient CRS/PASAM, OIM, PRODAF et GRET». La majorité de ces groupements a un compte auprès de la mutuelle Yarda ou ASUSU qui sont des institutions de micro finance locale.

Il est à retenir que tous les membres interrogés ici sont des femmes membres des dix groupements reconnus officiellement au niveau de la Mairie de Matamèye. L'idée est de voir si réellement ces actrices de développement font parties intégrantes des actions menées. Ces chiffres en disent assez. D'après même nos renseignements, même ceux qui ne sont pas encore reconnus au niveau de la commune sont composés des femmes. Ici 
donc, la première variable « intégration du genre » est prise en compte dans le jargon du développement communal.

Après l'identification, nous nous sommes intéressés à l'aspect reconnaissance juridique des groupements et la possession d'un compte bancaire, gage d'une certaine crédibilité auprès des partenaires.

Tableau 01 : Reconnaissance juridique des groupements et compte bancaire.

Groupements

Groupement 01

Groupement 02

Groupement 03

Groupement 04

Groupement 05

Groupement 06

Groupement 07

Groupement 08

Groupement 09

Groupement 10

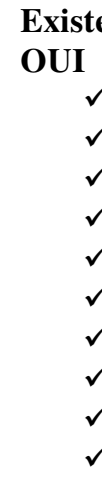

Source : notre enquête du 12 et 20 Août 2017
Existence compte d'épargne

OUI

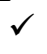

$v$
$v$
$v$

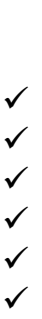

Il ressort clairement de ce tableau que tous les dix (10) groupements interrogés ont une reconnaissance officielle au niveau de la Mairie. Il s'agit d'un agrément à vie de la structure tant que celle-ci ne se détourne pas de ses activités d'origine. C'est donc un acte qui matérialise l'existence du groupement dans la commune et sa délivrance n'est pas très chère car délivrée à 5000 FCFA.

En outre sept (07) groupements sur les dix (10) ont un compte bancaire logé surtout au niveau des institutions de micro finance comme ASSUSU et YARDA qui ont des représentations à Matamèye. Le fait d'avoir un compte bancaire est d'une certaine notoriété car non seulement cela est connu de tous les membres mais aussi, les partenaires techniques et financiers se sentent plus responsables de faire un virement bancaire que de remettre des sommes colossales à la main. En principe les dépenses sont contrôlées car les sorties se font sur ordre de la présidente dudit groupement et les mouvements de fonds sont facilement vérifiables au niveau des mutuelles.

Les groupements identifiés, en plus de l'enregistrement officiel et de la disponibilité d'un compte, nous nous sommes intéressés aussi à l'aspect organisation du groupement. Il est important de souligner que pour être choisi en tant que membre, il faut "être actif et remarqué ». Cela veut dire qu'il faut bien travailler pour le groupement en fonction des taches qui vous sont dévolues. Enfin, il faut noter que c'est le groupement par la contribution de ses membres qui cotisent pour la mise en place d'un fonds initial et cela permet aux bailleurs de s'intéresser davantage. 
Il est à souligner aussi d'après nos recherches, nous avons constaté aussi que cinq (05) groupements font leur réunion de façon mensuelle entre les membres, quant aux cinq autres, ils la font de façon hebdomadaire. Cela démontre que ces groupements fonctionnent car les réunions entre les membres sont des aspects très fondamentaux au niveau d'un groupement. Les réunions permettent de faire le point sur la vie du groupement, l'état du programme en cours, la situation financière et éventuellement les difficultés du groupement.

Il faut noter que tous ces groupements ont des noms locaux à savoir:

Rahama, Hadin kai, Tallafi, Faraha, Takaici, Hadin gouywa, Marhaba, Taimakon jama'a, Dadin kowa et Albarka. Tous ces groupements sont localisés dans la commune urbaine de Matamèye. Quelles sont les principales activités de ces groupements ?

Tableau 02 : Les principales activités des groupements

Groupement

Groupement 01

Groupement 02

Groupement 03

Groupement 04

Groupement 05

Groupement 06

Groupement 07

Groupement 08

Groupement 09

Groupement 10
Activités exercées

Extraction d'huile

Warrantage

Embouche /Habbanayé

Fabrication de garin yara Jardinage

Warrantage

Extraction d'huile

Warrantage

Extraction d'huile

Extraction d'huile

Source : notre enquête du 12 et 20 Août 2017

On peut comprendre qu'à l'issue de ce tableau que les principales activités de ces groupements sont: "garin yara, savon traditionnel, spaghetti traditionnel, jardinage, warrantage, embouche, achat et stockage du mil ou du niébé, etc. », ces propos confirment ceux du Maire cité ci-haut.

Selon les propos des personnes interrogés, ces activités « ont permis aux femmes de la commune d'enregistrer un changement positif sur le plan économique, social et politique et surtout dans la promotion du genre ». En effet, toujours selon, ces membres, «ils n'ont pas besoin d'attendre la famille pour faire face à leurs dépenses depuis l'existence de ces groupements, d'ailleurs ils contribuent en partie aux dépenses de la famille ».

Ce qui n'est pas négligeable dans leur cas, car arriver à se prendre en charge dans ces temps-ci, c'est une aubaine. Ils continuent en nous disant que «les femmes commencent à comprendre l'intérêt d'un groupement féminin et demandent à adhérer ou à créer les leurs. Ces groupements ont un caractère très social ajoutent-elles car dès qu'un membre a une cérémonie 
(baptême, mariage ou décès), les autres lui viennent au secours et la vie est faite ainsi. »

\section{Des différences avec l'ordre ancien}

Pour avoir une idée plus poussée sur l'ordre actuel de la décentralisation, nous avons voulu en faire une comparaison ou du moins savoir s'il y'a des différences avec l'ordre ancien, c'est-à-dire au moment du système de la déconcentration. Selon nos interlocutrices, chaque système a ses qualités et défauts. Il y'en a des femmes qui ont profité en son temps maintenant elles ne se retrouvent pas. Ce qui est sûr c'est qu'avec ce nouveau système, le Maire actuel et son équipe font tout, pour associer les groupements féminins en fonction du partenaire en présence. "Ce ne sont pas tous les groupements qui sont invités à la fois à la Mairie, mais c'est en fonction de votre interlocuteur que vous êtes sollicités ». "Et si vous êtes appelés, vous ne serez pas déçus » affirment les femmes. D’autres soulignent le problème de doublon que l'on constate : la Mairie ou le Département peut appeler votre groupement le même jour pour la même action. On a l'impression de fois qu'ils n'arrivent pas à se comprendre. Il en est de l'octroi de l'agrément. La mairie tout comme le département en délivre sans en spécifier les compétences. Il est clair que la décentralisation territoriale qui est un processus d'aménagement de l'État unitaire qui consiste à transférer des compétences administratives de l'État vers des entités (ou des collectivités) locales distinctes de lui, est en marche, et les actions de développement intègrent aussi la composante féminine comme en témoigne le cas de Matamèye.

Il s'agit d'un long processus, où à chaque étape, les nostalgiques de l'un ou l'autre système, chacun essaie de tirer la couverture vers lui. Au centre, il y a les citoyens qui attendent la réalisation de ce mot magique qu'est leur développement local, nous serons tentés de le dire.

\section{Conclusion}

Le développement local amorcé par nos Etats et poursuivi par nos communes dans un contexte de décentralisation est un processus de longue haleine. Il passe nécessairement par la prise en compte véritable de la composante féminine qui constitue un maillon non moins important de la société.

Au cours de notre étude, nous nous sommes intéressés à la promotion du genre et de l'équité dans toutes les actions de développement contenues dans le PDC Matamèye 2012-2016. Pour ce faire, notre document de travail a été le PDC de la commune ainsi que les outils élaborés pour la collecte des données complémentaires afin de trianguler les informations. 
A l'issue des différents entretiens conduits avec les acteurs clés ainsi que l'exploitation du document de PDC de la commune, les résultats nous ont permis de confirmer notre hypothèse : les actions du développement sont déterminées par la promotion et l'équité entre le genre. La commune a favorisé la création des groupements féminins et leur mise en relation avec les projets et ONGs intervenant dans la localité. Ces groupements exercent des activités génératrices de revenus qui sont d'une grande utilité non seulement pour les promotrices mais également pour la commune de façon générale.

\section{References:}

1. ACT-Consults. 2000. Comment mieux articuler les appuis aux initiatives de développement à la base et les appuis à la décentralisation ? Paris: Ministère des Affaires étrangères.

2. Antoine Goxe, 2006 «Gouvernance territoriale et développement durable: implications théoriques et usages rhétoriques »pp. 151-170, in : R. Pasquier, V. Simoulin, J. Weisbein,

3. Éds, La gouvernance territoriale. Pratiques, discours et théories, Paris, LGDJ, Tome 44, coll. «Droit et Société ».

4. Bako-Arifari Nassirou et Laurent Pierre Joseph (dir.). 1998. Les dimensions sociales et économiques du développement local et la décentralisation en Afrique au Sud du Sahara. Bulletin APAD, (15).

5. Bierschenk Thomas, et Jean-Pierre Olivier de Sardan (dir.). 1998. Les pouvoirs au village : le Bénin rural entre démocratisation et décentralisation. Paris: Karthala.

6. Bernard Guesnier, Développement local et décentralisation, Genève, Ed. Région Europe, 1986.

7. Code général des collectivités territoriales du Niger, 2011.

8. Diop Amadou, 2009, Développement local, gouvernance territoriale - Enjeux et perspectives, Ed. Karthala, 230p.

9. Ferguène Améziane, 2005, Gouvernance locale et développement territorial - Le cas des pays du sud, Paris, l'Harmattan. 32p.

10. Hubert Coudrieux, 1988, La science des systèmes et des exploitations agricoles, Ed. Universitaires, UNMFREO, 1990.

11. Jean-Christophe DEBERRE (2007), Afrique contemporaine $\left(\mathrm{n}^{\circ}\right.$ 221), Éditeur : De Boeck Supérieur, pp 45-54.

12. Mahaman ABA, <<processus de planification communale au Niger: démarche, acteurs impliques et difficultés ». Working paper n342009.

13. Maurice Blanc, 1992, « La transaction sociale », Ed. L'Harmattan, Paris. 
14. Michel Bouvier, 1986 : « Solidarités locales: les chartes intercommunales de développement et d'aménagement », (sous la direction de), Paris, Libraire générale de droit et de jurisprudence, 1986.

15. Mohamed HADDAD et Mohamed Amir BOUZAIDA, Développement local et gouvernance territoriale : Enjeux et perspectives post-révolution, in Journal of Academic Finance (J.A.F.) ISSN 1923-2993, fall 2013.

16. http://www.oneworld.org/ecdpm/pubs/wp58_fr.htm 